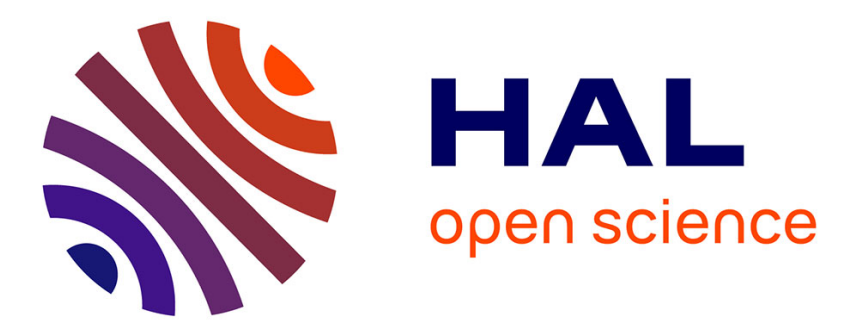

\title{
DIifferent origins or different evolutions? Decoding the spectral diversity among C-type asteroids
}

P. Vernazza, J. Castillo-Rogez, P. Beck, J. Emery, R. Brunetto, M. Delbò, M. Marsset, F. Marchis, O. Groussin, B. Zanda, et al.

\section{- To cite this version:}

P. Vernazza, J. Castillo-Rogez, P. Beck, J. Emery, R. Brunetto, et al.. DIifferent origins or different evolutions? Decoding the spectral diversity among C-type asteroids. The Astronomical Journal, 2017, 153 (2), 10.3847/1538-3881/153/2/72 . hal-01790534

\section{HAL Id: hal-01790534 \\ https://hal.science/hal-01790534}

Submitted on 13 May 2018

HAL is a multi-disciplinary open access archive for the deposit and dissemination of scientific research documents, whether they are published or not. The documents may come from teaching and research institutions in France or abroad, or from public or private research centers.
L'archive ouverte pluridisciplinaire HAL, est destinée au dépôt et à la diffusion de documents scientifiques de niveau recherche, publiés ou non, émanant des établissements d'enseignement et de recherche français ou étrangers, des laboratoires publics ou privés. 


\title{
DIFFERENT ORIGINS OR DIFFERENT EVOLUTIONS? DECODING THE SPECTRAL DIVERSITY AMONG C-TYPE ASTEROIDS
}

\author{
P. Vernazza ${ }^{1}$, J. Castillo-Rogez ${ }^{2}$, P. Beck ${ }^{3}$, J. Emery ${ }^{4}$, R. Brunetto ${ }^{5}$, M. Delbo ${ }^{6}$, M. Marsset ${ }^{1}$, F. Marchis $^{7}$, O. Groussin ${ }^{1}$, \\ B. Zanda ${ }^{8,9}$, P. Lamy ${ }^{1}$, L. Jorda ${ }^{1}$, O. Mousis ${ }^{1}$, A. Delsanti ${ }^{1}$, Z. Djouadi ${ }^{5}$, Z. Dionnet ${ }^{5}$, F. Borondics ${ }^{10}$, And B. Carry ${ }^{6}$ \\ ${ }^{1}$ Aix Marseille Univ, CNRS, LAM, Laboratoire d'Astrophysique de Marseille, Marseille, France; pierre.vernazza@lam.fr \\ 2 Jet Propulsion Laboratory, California Institute of Technology, 4800 Oak Grove Drive, Pasadena, CA 91109, USA \\ ${ }^{3}$ UJF-Grenoble 1, CNRS-INSU, Institut de Planétologie et d'Astrophysique de Grenoble (IPAG), UMR 5274, Grenoble F-38041, France \\ ${ }^{4}$ Department of Earth and Planetary Sciences and Planetary Geosciences Institute, University of Tennessee, Knoxville, TN 37996-1410, USA \\ ${ }^{5}$ Institut d'Astrophysique Spatiale, CNRS, UMR-8617, Université Paris-Sud, bâtiment 121, F-91405 Orsay Cedex, France \\ ${ }^{6}$ Laboratoire Lagrange, UNS-CNRS, Observatoire de la Cote d'Azur, Boulevard de l'Observatoire-CS 34229, F-06304 Nice Cedex 4, France \\ ${ }^{7}$ Carl Sagan Center at the SETI Institute, Mountain View, CA 94043, USA \\ ${ }^{8}$ IMCCE, Observatoire de Paris, 77 avenue Denfert-Rochereau, F-75014 Paris Cedex, France \\ ${ }^{9}$ Institut de Mineralogie, de Physique des Materiaux, et de Cosmochimie (IMPMC), Sorbonne Universites, \\ Museum National d'Histoire Naturelle, UPMC Universite Paris 06, UMR CNRS 7590, IRD UMR 206, 61 rue Buffon, F-75005 Paris, France \\ ${ }^{10}$ SMIS Beamline, Soleil Synchrotron, BP48, L’Orme des Merisiers, F-91192 Gif sur Yvette Cedex, France \\ Received 2016 November 10; revised 2016 December 3; accepted 2016 December 5; published 2017 January 16
}

\begin{abstract}
Anhydrous pyroxene-rich interplanetary dust particles (IDPs) have been proposed as surface analogs for about twothirds of all C-complex asteroids. However, this suggestion appears to be inconsistent with the presence of hydrated silicates on the surfaces of some of these asteroids, including Ceres. Here, we report the presence of enstatite (pyroxene) on the surface of two C-type asteroids (Ceres and Eugenia) based on their spectral properties in the mid-infrared range. The presence of this component is particularly unexpected in the case of Ceres, because most thermal evolution models predict a surface consisting of hydrated compounds only. The most plausible scenario is that Ceres' surface has been partially contaminated by exogenous enstatite-rich material, possibly coming from the Beagle asteroid family. This scenario questions a similar origin for Ceres and the remaining C-types, and it possibly supports recent results obtained by the Dawn mission (NASA) that Ceres may have formed in the very outer solar system. Concerning the smaller $D \sim 200 \mathrm{~km} \mathrm{C}$-types such as Eugenia, both their derived surface composition (enstatite and amorphous silicates) and low density $\left(<1.5 \mathrm{~g} \mathrm{~cm}^{-3}\right)$ suggest that these bodies accreted from the same building blocks, namely chondritic porous, pyroxene-rich IDPs and volatiles (mostly water ice), and that a significant volume fraction of these bodies has remained unaffected by hydrothermal activity likely implying a late accretion. In addition, their current heliocentric distance may best explain the presence or absence of water ice at their surfaces. Finally, we raise the possibility that CI chondrites, Tagish-Lakelike material, or hydrated IDPs may be representative samples of the cores of these bodies.
\end{abstract}

Key words: meteorites, meteors, meteoroids - methods: data analysis - methods: laboratory: solid state methods: observational - minor planets, asteroids: general - techniques: spectroscopic

\section{INTRODUCTION}

C-complex asteroids (B, C, Cb, Cg, Cgh, Ch; DeMeo et al. 2009), which constitute the main population in the asteroid belt (they represent $\sim 38 \%$ of the mass of the belt when Ceres, Pallas, Vesta, and Hygeia are excluded; 66\% otherwise; DeMeo \& Carry 2013), have been extensively studied via spectroscopic measurements in the visible and near-infrared range $(0.4-4 \mu \mathrm{m}$; Vilas \& Gaffey 1989; Hiroi et al. 1993, 1996; Vilas et al. 1993; Rivkin et al. 2003; Clark et al. 2010; Rivkin 2012; Takir \& Emery 2012; Vernazza et al. 2015, 2016) and to a lesser extent in the mid-infrared domain (7-13 $\mu \mathrm{m}$ range; Barucci et al. 2002; Milliken \& Rivkin 2009; Vernazza et al. 2015; Marsset et al. 2016). Overall, these observations have provided the following constraints.

(a) Ch- and Cgh-type asteroids appear to be the parent bodies of the most common type of hydrated meteorites, namely CM chondrites (Vilas \& Gaffey 1989; Vilas et al. 1993; Rivkin 2012; Lantz et al. 2013; Burbine 2014, pp. 365-415; McAdam et al. 2015; Rivkin et al. 2015; Vernazza et al. 2016). Furthermore, it appears—on the basis of a spectral comparison in the visible and nearinfrared range-that $\mathrm{CM}$ chondrites match both the surface and interior of $\mathrm{Ch}$ - and Cgh-type asteroids implying a homogeneous internal structure for these bodies (Vernazza et al. 2016). Finally, Ch- and Cgh-type asteroids represent the vast majority of the $\mathrm{C}$-complex asteroids displaying a sharp spectral feature in the $3 \mu \mathrm{m}$ region (Takir \& Emery 2012).

(b) The surface material of B-, C-, Cb-, and Cg-type asteroids (BCG asteroids hereafter; $40 \%$ of mass of the belt-see DeMeo \& Carry 2013) appears to be mostly absent from meteorite collections (see the discussion in Vernazza et al. 2015). Instead, interplanetary dust particles (IDPs) may be samples of these objects (Vernazza et al. 2015; Marsset et al. 2016). Specifically, Vernazza et al. (2015) have opened the possibility that pyroxene-rich chondritic porous IDPs (which in fact contain more than 50\% amorphous material; Bradley 1994; Ishii et al. 2008) may be the closest analogs to the surfaces of these objects. However, observations of these bodies in the $3 \mu \mathrm{m}$ region have shown that not all surfaces are compatible with anhydrous silicates as major surface components (e.g., Rivkin et al. 2003; Takir \& Emery 2012; De Sanctis et al. 2015, 2016). Some of these objects (e.g., Ceres) display hydrated silicates at their surfaces (e.g., Rivkin 
et al. 2003; Takir \& Emery 2012) as well as carbonates in the case of Ceres (Rivkin et al. 2006; De Sanctis et al. 2015, 2016), minerals that are clearly inconsistent with chondritic porous (anhydrous) IDPs. Observations of these bodies in the $3 \mu \mathrm{m}$ region also suggest that their surfaces may not only consist of a refractory phase but they may also include volatiles such as water ice (Campins et al. 2010; Rivkin \& Emery 2010; Takir \& Emery 2012; Combe et al. 2016a, 2016b). In summary, whereas a new interpretation of the surface composition of BCG-types has emerged (e.g., pyroxene-rich IDPs), it does not appear to always be consistent with the spectral diversity observed within this vast group of bodies.

To make progress in our understanding of the surface composition of BCG-type asteroids, we explore the spectral properties of two of them: (1) Ceres and (45) Eugenia in the mid-infrared, over the 5-35 $\mu \mathrm{m}$ range. These objects were chosen to investigate, to some extent, the spectral diversity observed in the $3 \mu \mathrm{m}$ region among C-type bodies as they represent two of the five " $3 \mu \mathrm{m}$ " classes. Four of these classes were defined by Takir \& Emery (2012), whereas the last one comprises objects with featureless spectra in this wavelength range. In recent years, it has been shown that mid-infrared spectroscopy is a powerful tool for constraining the surface composition of asteroids of low thermal inertia (i.e., high surface porosity), as their spectra display distinct emissivity features (e.g., Emery et al. 2006; Vernazza et al. 2011, 2012, $2013,2015)$. These features can then be used to determine (A) the relative abundance of the main minerals present on the object and/or (B) the meteoritic/IDP analog. Case (A) can be implemented using a spectral decomposition model (e.g., Vernazza et al. 2012, 2015; Marsset et al. 2016) whereas case (B) requires using transmission infrared spectroscopic measurements of IDPs and meteorites (e.g., Sandford \& Walker 1985; Izawa et al. 2010; Vernazza et al. 2012; Beck et al. 2014; Merouane et al. 2014). Importantly, sampling the full $7-25 \mu \mathrm{m}$ range is necessary in order to properly assess the surface composition of an asteroid on the basis of mid-infared spectroscopic observations, provided that its spectrum displays distinct emissivity features (Vernazza et al. 2012). Limiting the compositional analysis to the $7-12 \mu \mathrm{m}$ range allows for the detection of crystalline silicates, such as enstatite and olivine, but it prevents the unambiguous detection of amorphous or hydrated silicates. For these latter components, observations at longer wavelengths $(12-25 \mu \mathrm{m}$ range) are required.

\section{OBSERVATIONS AND THERMAL MODELING}

\subsection{Observations and Data Reduction}

New mid-infrared spectral measurements of (1) Ceres in the $\sim 5-37 \mu \mathrm{m}$ range were obtained using FORCAST, a dualchannel mid-infrared camera and spectrograph, mounted at the Nasmyth focus of the $2.5 \mathrm{~m}$ telescope of the Stratospheric Observatory for Infrared Astronomy (SOFIA). The observing run was conducted on 2015 June 5 on a flight originating from Palmdale (California, USA). Details of the SOFIA observations are summarized in Table 1.

The FORCAST spectrograph with a $2.4 \times 194$ arcsec slit and a suite of four grisms $(4.9-8.0 \mu \mathrm{m}, 8.4-13.7 \mu \mathrm{m}$, 17.6-27.7 $\mu \mathrm{m}$, and $28.7-37.1 \mu \mathrm{m})$ was used in the lowresolution prism mode $(R=\lambda / \Delta \lambda \approx 200)$ for acquisition of
Table 1

Observing Parameters of Our SOFIA Observations

\begin{tabular}{llccc}
\hline \hline Asteroid & Observing Date & $r^{\mathrm{a}}(\mathrm{au})$ & $\Delta^{\mathrm{b}}(\mathrm{au})$ & $\alpha^{\mathrm{c}}\left({ }^{\circ}\right)$ \\
\hline 1 Ceres & 2015 Jun 05 & 2.92 & 2.22 & 16.5 \\
\hline
\end{tabular}

Notes.

${ }^{\mathrm{a}} r$ is the heliocentric distance.

b $\Delta$ is the SOFIA-asteroid distance.

${ }^{\mathrm{c}} \alpha$ is the phase angle.

the spectra in the $\sim 5-37 \mu \mathrm{m}$ wavelength range. Images in four filters $(6.6,11.1,19.7$, and $34.8 \mu \mathrm{m})$ were also secured.

Concerning the data reduction, the SOFIA Science Center provides the processed, flux calibrated Level 3 products for the FORCAST imaging and grism modes. A detailed description of the data reduction steps can be found in the Guest Investigator Handbook for FORCAST Data Products (https://www. sofia.usra.edu/sites/default/files/FORCAST_GI_Handbook_ RevB.pdf).

Finally, we complemented our data set with a Ceres' spectrum retrieved from the ISO archive (http://www.cosmos.esa.int/ web/iso/access-the-archive) and with a Spitzer emissivity spectrum for (45) Eugenia taken from Marchis et al. (2012).

\subsection{Modeling of the Thermal Emission}

We modeled the thermal emission of Ceres in order to derive its infrared emissivity using the Near Earth Asteroid Thermal model (NEATM) developed by Harris (1998), which assumes a spherical body. The NEATM has two free parameters: the radius of the body $r_{\mathrm{n}}$ and the beaming factor $\eta$, which accounts for the combined effect of roughness, thermal inertia, and geometry (pole orientation, phase angle). We assumed a bond albedo in the visible wavelength range of 0.034 (Li et al. 2016). We computed the temperature distribution at the surface of Ceres for the ISO and SOFIA observing dates (Table 1), from which we derived the Spectral Energy Distribution (SED). Finally, the infrared emissivity was obtained by dividing the observational data by the SED.

The radius $r_{\mathrm{n}}$ controls the intensity of the SED, whereas $\eta$ controls its intensity and shape and consequently the slope of the infrared emissivity. We adjusted these two parameters in order to obtain a consistent solution between the ISO and SOFIA data in terms of radius, beaming factor, and infrared emissivity. For ISO, we obtained a radius of $471 \mathrm{~km}$ and a beaming factor of 0.90 , and for SOFIA a radius of $468 \mathrm{~km}$ and a beaming factor of 0.93 . The radii are in excellent agreement with Ceres' mean radius of $\sim 470 \mathrm{~km}$ (Park et al. 2016; Russell et al. 2016), whereas the beaming factor close to 1 is consistent with the low thermal inertia of Ceres estimated to be less than $15 \mathrm{~J} / \mathrm{m}^{2} / \mathrm{s}^{1 / 2} / \mathrm{K}$ by Chamberlain et al. (2009) and in the $5-25 \mathrm{~J} / \mathrm{m}^{2} / \mathrm{s} / 2 / \mathrm{K}$ range by Mueller \& Lagerros (1998). The ISO and SOFIA data, the modeled SEDs and the corresponding infrared emissivity of Ceres are shown in Figure 1. We improved the signal-to-noise ratio $(\mathrm{S} / \mathrm{N})$ of the emissivity by binning the ISO and SOFIA data. Since the ISO data have a better $\mathrm{S} / \mathrm{N}$ than the SOFIA data, we used the ISO data whenever available, and the SOFIA data to only fill the ISO spectral gaps. However, we kept the SOFIA data in the $22-27 \mu \mathrm{m}$ range, where the flux is the highest and where it overlaps the ISO data, to provide consistency between the ISO and SOFIA data sets. 


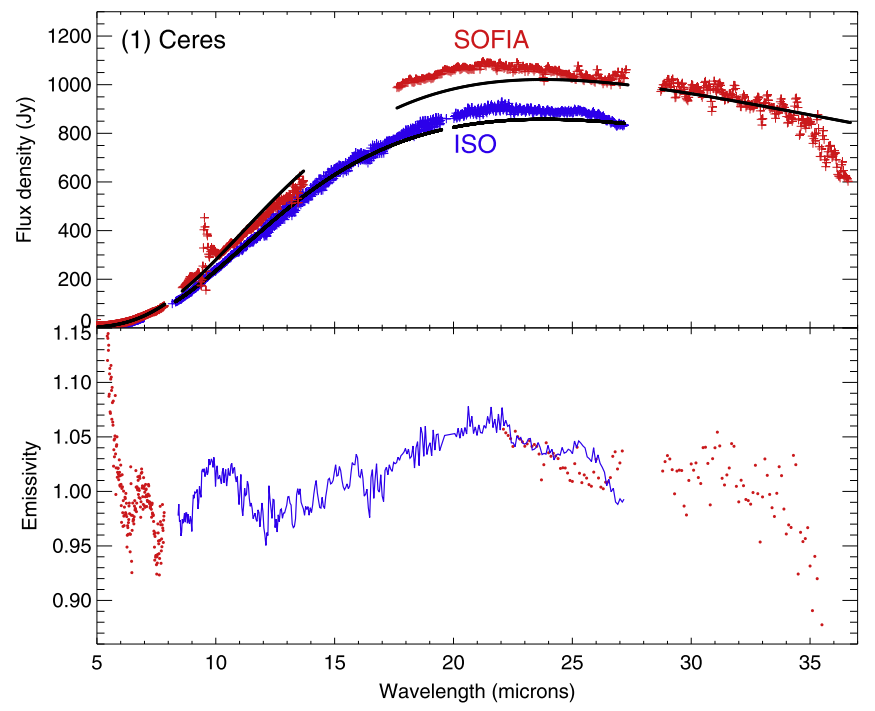

Figure 1. Upper panel: the SED of (1) Ceres obtained by ISO (blue curve) and SOFIA (red curve) with the best-fit thermal models (black curves). Lower panel: emissivity spectrum of (1) Ceres with the same color-coding as above.

\section{RESULTS}

In this section, we present our analysis of the surface composition of (1) Ceres and (45) Eugenia using the two approaches (case A and case B) mentioned in the Introduction.

\section{1. (1) Ceres}

In a first step, we performed a basic reconnaissance of the emissivity features present in the Ceres spectrum and found three features centered at $\sim 7, \sim 10$, and $\sim 22 \mu \mathrm{m}$ that stand out in both the SOFIA and ISO spectra. Whereas the simultaneous presence of the $\sim 10$ and $\sim 22 \mu$ m features is coherent with the presence of phyllosilicates such as those seen in CI and CM chondrites (e.g., Vernazza et al. 2012; Beck et al. 2014), the $\sim 7 \mu \mathrm{m}$ feature suggests the presence of carbonates at the surface, consistent with previous observations (Rivkin et al. 2006; De Sanctis et al. 2015). Note that ammonium cations within ammoniated phyllosilicates also show a $6.9 \mu \mathrm{m}$ emissivity feature (Petit et al. 1999). Ceres' $\sim 7 \mu \mathrm{m}$ feature may therefore not only be due to the presence of carbonates at the surface but also to that of ammoniated phyllosilicates (De Sanctis et al. 2015). However, the 3.95 feature observed in Ceres' near-infrared spectrum can only be attributed to carbonates because $\mathrm{NH}_{4}{ }^{+}$products do not possess such a spectral band.

In a second step, we performed a detailed comparison of the emissivity spectrum of (1) Ceres with a set of mid-infrared spectroscopic measurements of KB-diluted meteorites (see Appendix A.1 and Figure 6). For a quantitative comparison of each meteorite to Ceres, we used the position and width of the diagnostic $\sim 7, \sim 10$, and $\sim 22 \mu \mathrm{m}$ emissivity features as sole criteria rather than the spectral amplitude. We thus allowed the spectral contrast of the meteorite spectrum to vary and minimized the rms difference between the two spectra.

These comparisons reveal that $\mathrm{CI}$ and $\mathrm{CM}$ chondrites as well as the ungrouped Tagish Lake meteorite provide the closest-yet unsatisfactory-spectral match to Ceres (Figure 2). Whereas the two most prominent features in the Ceres spectrum at $\sim 10$ and $\sim 22 \mu \mathrm{m}$ are also seen in the meteorite spectra and the weaker feature at $\sim 7 \mu \mathrm{m}$ is further seen in some

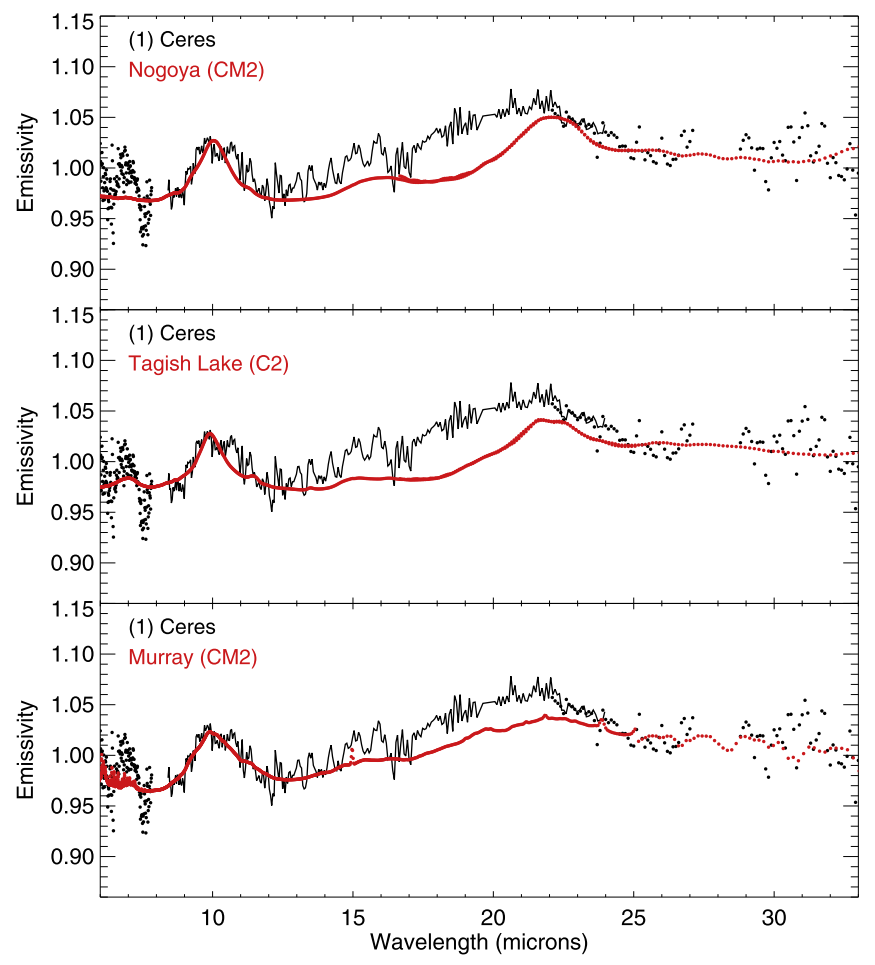

Figure 2. Comparison between the emissivity spectrum of (1) Ceres and the spectra of three carbonaceous chondrite meteorites. Whereas the two most prominent emissivity features in the Ceres spectrum (at $\sim 10 \mu \mathrm{m}$ and $\sim 22 \mu \mathrm{m}$ ) are also seen in the meteorite spectra, their shape and profile appear quite different in both regions. Note that the mismatch in the $10 \mu \mathrm{m}$ region is by far the more decisive with hydrated meteorites being single peaked whereas Ceres is double peaked. This spectral difference suggests that an additional component is present at the surface of Ceres. Finally, an $\sim 7 \mu \mathrm{m}$ carbonate feature is observed both in the Ceres and meteorite spectra (especially in the Tagish Lake spectrum).

of them (especially in the Tagish Lake spectrum), the features in the meteorite spectra (in particular in the $10 \mu \mathrm{m}$ region) reproduce only partially the spectrum of Ceres. The $10 \mu \mathrm{m}$ band of all meteorites exhibit a single peak centered at $\sim 10 \mu \mathrm{m}$ whereas that of Ceres is double peaked with maxima at $\sim 9.8$ and $\sim 10.65 \mu \mathrm{m}$. In the $20 \mu \mathrm{m}$ region, the wide emission band appears narrower in the meteorite spectra than in the Ceres spectrum. Finally, the intensity of the $7 \mu \mathrm{m}$ band is not well reproduced, implying more carbonates at the surface of Ceres than within the carbonate-rich Tagish Lake meteorite $(\sim 12 \%$ by volume; Bland et al. 2004) and/or the presence of ammoniated phyllosilicates at the surface of Ceres. These discrepancies observed in the 10 and $20 \mu \mathrm{m}$ regions open the possibility for at least an additional component at the surface of Ceres that could well be enstatite (a type of pyroxene). Indeed, the spectrum of Ceres in this wavelength region is remarkably similar to that of (10) Hygeia (see Appendix A.2 for a discussion and Figure 7) whose spectrum has been well reproduced assuming enstatite as the dominant surface mineral (Vernazza et al. 2015).

To investigate this possibility, we used a spectral decomposition model as presented in Vernazza et al. (2015) and Marsset et al. (2016). First, the emissivity of enstatite $\left(\mathrm{Mg}_{2} \mathrm{Si}_{2} \mathrm{O}_{6}\right)$ was calculated for a distribution of hollow spheres with small sizes $(<1 \mu \mathrm{m})$ compared to wavelength (Min et al. 2003). The optical constants ( $\mathrm{n}$ and $\mathrm{k}$ ) of enstatite were retrieved from the Jena database (http://www.astro.uni-jena.de/Laboratory/Database/ databases.html). Second, we calculated the best fit to the Ceres 


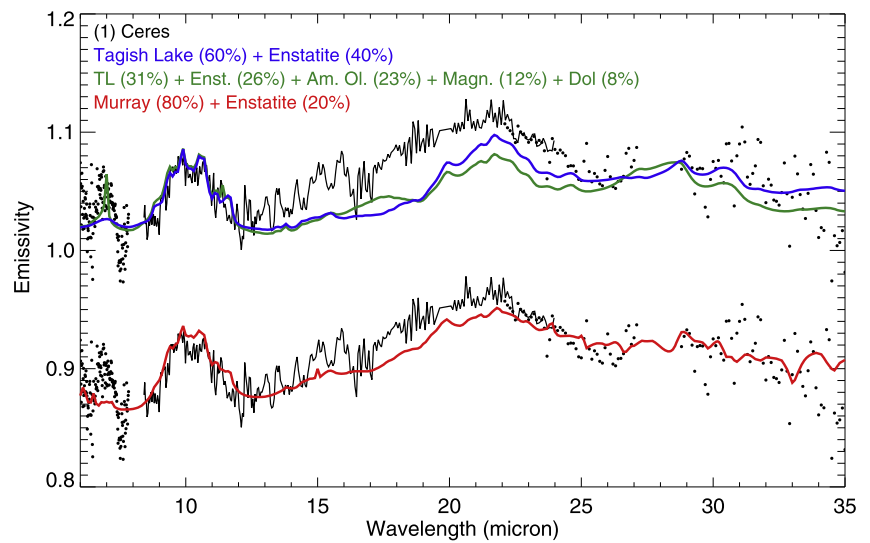

Figure 3. Comparison between the spectrum of (1) Ceres and the two best-fit models. These models (blue and red curves) result from the linear combination of the emissivity spectra of enstatite and $\mathrm{CI} / \mathrm{CM}$ chondrites (here the CM2 chondrite Murray and the CI/CM Tagish Lake). We also display an additional best-fit model (green curve) to highlight that additional components such as amorphous silicates of olivine-like composition and magnetite may also be present at the surface of Ceres (see the text). Of prime importance, the best fit requires at least $20 \%$ enstatite in the linear mixture.

spectrum resulting from the linear combination of the emissivity spectra of enstatite and $\mathrm{CI} / \mathrm{CM}$ chondrites as well as Tagish Lake. Examples of the two best fits are highlighted in Figure 3. The addition of enstatite not only improves the match in the $10 \mu \mathrm{m}$ region (i.e., the chi-square is reduced by a factor of two with respect to a best fit with Tagish Lake only) but it also helps to improve the fit in the $20 \mu \mathrm{m}$ region by better reproducing the width of the emission peak. Additional minerals were tested (crystalline olivine, diposide, amorphous silicates) but none of them reproduced the spectrum of Ceres. In particular, an increasing fraction of crystalline olivine or diopside led to a steady increase of the chi-square value justifying the rejection of these components on Ceres' surface in amounts larger than a few percent. A priori, we cannot exclude the presence of minor amounts $(<20 \%)$ of amorphous silicates (olivine and pyroxene) at the surface of Ceres as these silicates possess very similar spectral properties to those of hydrated silicates in the $10 \mu \mathrm{m}$ region. However, their spectral properties in the $20 \mu \mathrm{m}$ region diverge from those of Ceres with emissivity peaks around $\sim 18 \mu \mathrm{m}$. Along these lines, we also cannot exclude the presence of minor amounts of iron fragments/particles at the surface, such components being spectrally featureless in the mid-infrared range. Also, the mid-infrared spectral properties of Ceres imply that magnetite can only be a minor $(<10 \%)$ surface component (Yang \& Jewitt 2010) since its emissivity spectrum shows a single peak in the $7-25 \mu \mathrm{m}$ region centered at $\sim 17 \mu \mathrm{m}$, which is at odds with the spectral properties of Ceres. Lastly, we calculated the best fit to Ceres' spectrum without enstatite, which was obtained with CI-, CM-, or Tagish-Lake-like material only (Figure 2), thus highlighting the unique contribution of enstatite to the improvement of the best fit.

In summary, the surface composition of Ceres appears to be dominated by (1) carbonates, (2) phyllosilicates (possibly ammoniated), and (3) enstatite. The presence of anhydrous material (enstatite) on a surface otherwise dominated by products of aqueous alteration (carbonates, phyllosilicates) was unexpected.

\section{2. (45) Eugenia}

Eugenia is a C-type asteroid with a mean diameter of $\sim 210 \mathrm{~km}$ (Marchis et al. 2012). Its mid-infrared spectrum is

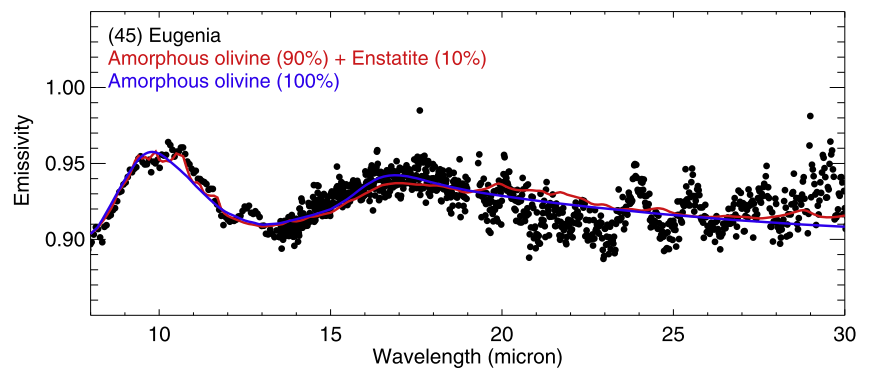

Figure 4. Comparison between the spectrum of (45) Eugenia and the two bestfit models. Based on the model, which incorporates enstatite (red curve), it appears that both amorphous and crystalline components are present on the surface of Eugenia. Furthermore, the crystalline components are mostly pyroxene-rich.

characterized by two prominent features centered at $\sim 10 \mu \mathrm{m}$ and $\sim 17 \mu \mathrm{m}$. The $10 \mu \mathrm{m}$ feature is remarkably similar in shape to that of Ceres, whereas the $17 \mu \mathrm{m}$ feature appears different from any feature observed so far in meteorite spectra. Further comparison of its emissivity spectrum with the spectroscopic measurements of $\mathrm{KBr}$-diluted meteorites reveals no convincing match, suggesting that Eugenia is unsampled by meteorites as already proposed by Vernazza et al. (2015). Importantly, the absence of a $\sim 22 \mu \mathrm{m}$ feature, as observed in the spectra of Ceres and hydrated meteorites, implies the absence of phyllosilicates on the surface of Eugenia. This conclusion is well supported by measurements at shorter wavelengths that revealed an absence of hydration band in the $3 \mu \mathrm{m}$ region (Rivkin et al. 2003).

A spectral decomposition model with the same minerals as in Vernazza et al. (2015) reproduces the spectral behavior of Eugenia with crystalline pyroxene (enstatite; $\sim 10 \%$ ) and amorphous silicates of olivine-like composition ( $\sim 90 \%)$ only (Figure 4). Note that the mid-infrared spectrum of amorphous silicates of pyroxene-like composition differs only slightly from the one of olivine-like composition. Therefore, the constraint on the mineralogy of the amorphous component is less compelling than for the crystalline one.

\section{DISCUSSION}

Our analysis of the surface composition of (1) Ceres and (45) Eugenia appears not only coherent with previous findings based on spectroscopic observations in the $3 \mu \mathrm{m}$ region (e.g., Rivkin et al. 2003, 2006; De Sanctis et al. 2015, 2016) but it is further complementary. Concerning Ceres, early observations by Rivkin et al. (2003) in the $3 \mu \mathrm{m}$ region showed that its surface is less hydrated than that of CM-like Ch- and Cgh-types (by $\sim 30 \%$ ) opening the possibility of the presence of an anhydrous component at its surface. Later on, carbonates were detected by Rivkin et al. (2006) and further confirmed by observations with the VIR spectrometer of the Dawn mission (De Sanctis et al. 2015, 2016), which further unambiguously identified phyllosilicates (De Sanctis et al. 2015). Enstatite, however, has not been previously reported as a main surface component for Ceres. Note that an additional dark and neutral component such as carbon may well be present at the surface (Hendrix et al. 2016) in order to explain the overall low reflectivity of Ceres (Bond Albedo 0.035; Li et al. 2016). Concerning Eugenia, Rivkin et al. (2003) found its surface to be essentially anhydrous. Our spectral analysis not only confirms this early result but also provides a more detailed 
characterization of the mineralogical composition of its surface (mixture of enstatite and amorphous silicates).

Hereafter, we first discuss the probable exogenous origin of enstatite on the surface of Ceres. We further underline that this "enstatite anomaly" is also observed in the case of Hygiea, the second largest $\mathrm{C}$-type asteroid. We next attempt to formulate a comprehensive scenario for the formation and evolution of IDP-like $D \sim 200 \mathrm{~km}$ C-type bodies. In particular, we introduce a new hypothesis for the composition of the putative cores of these bodies. In this respect, hydrated extraterrestrial materials such as CI chondrites, the Tagish Lake meteorite, and hydrous IDPs appear as plausible candidates.

\subsection{On the Origin of the Presence of Enstatite on the Surface of Ceres}

\subsubsection{Enstatite as an Endogenous Component}

A first explanation for the presence of enstatite on the surface of Ceres is that such material was part of the primordial building blocks of Ceres along with water ice and that a fraction of this material was preserved intact during Ceres' early thermal evolution. Up to now, however, none of the numerical models simulating this evolution (e.g., McCord \& Sotin 2005; Castillo-Rogez \& McCord 2010; Castillo-Rogez 2011; Neveu \& Desch 2015) have produced a compositional stratification that would naturally lead to the present surface being a mixture of hydrous and primordial anhydrous silicates following impact mixing. Most simulations (e.g., McCord \& Sotin 2005; Castillo-Rogez \& McCord 2010), which assume that Ceres accreted from a mixture of ice and anhydrous material in roughly equal proportions in agreement with the Dawn-derived density estimate $\left(2.16 \mathrm{~g} \mathrm{~cm}^{-3}\right.$; Park et al. 2016; Russell et al. 2016), predict that the primordial crust would have foundered as a consequence of global melting and impacting, which has been simulated by Formisano et al. (2016). Following these models, the present crust should entirely consist of products of aqueous alteration. Note that one of the models by McCord \& Sotin (2005) assumed an intact$130 \mathrm{~km}$ thick-primordial anhydrous outer shell (their case 1) superimposed on a hydrated, phyllosilicate-rich core. This scenario seems unlikely because it is highly improbable that impacts over the course of the solar system history could have mixed the two layers and produced the homogeneity of the hydrated material found on the surface (Ammannito et al. 2016).

Finally, we discuss hereafter two scenarios that seem highly unlikely to explain the coexistence of anhydrous and hydrous silicates at the surface of Ceres.

(a) It is unlikely that the anhydrous silicates could originate from the dehydration of the phyllosilicates following micrometeorite bombardment. First, micrometeorite impacts do not produce enough energy to bring the surface temperature to the required $>500^{\circ}$. C temperature (King et al. 2015). Second, this effect would also apply to many other asteroids, such as Pallas and/or CM-like bodies, yet this is not supported by observations.

(b) It also seems unlikely that silicates would be subject to partial aqueous alteration in Ceres' near surface. This scenario implies a dearth of water. However, the abundance of $\mathrm{Mg}$-serpentine (antigorite; De Sanctis et al. 2015) implies advanced alteration leading to the leaching of iron from the original anhydrous silicates.
Furthermore, gravity and topography observations point to an abundance of volatiles at depth (Fu et al. 2016).

\subsubsection{Enstatite as an Exogenous Component}

A more plausible explanation for the presence of enstatite on the surface of Ceres is impact implantation of exogenous material. Prior works have demonstrated that impactors can survive during hypervelocity collisions with porous targets and thus remain intact at their surfaces (Daly \& Schultz 2015, 2016; Avdellidou et al. 2016; Turrini et al. 2016). Retention of exogenous material may not only work for porous targets but for compact ones as well. Indeed, the presence of dark material on Vesta's surface (McCord et al. 2012; Reddy et al. 2012; Turrini et al. 2014) has been interpreted as direct evidence for projectile retention. A recent study also raised the possibility that the presence of olivine at several locations on Vesta's surface (Ammannito et al. 2013; Ruesch et al. 2014; Palomba et al. 2015; Poulet et al. 2015) may be best explained as impact contamination (Turrini et al. 2016).

From above, the hypothesis that impact-delivered debris could have been retained on Ceres appears plausible and Ceres' present surface composition may therefore not only reflect its endogenic evolution but also that of exogenous compounds (Daly \& Schultz 2015). In this scenario, it remains to be understood whether the exogenic products $(20 \%-40 \%$ volume fraction following our spectral analysis) essentially originated from a few large impacts or from numerous smaller contributions by IDPs. The first case (a few large impacts) seems unlikely at first glance if one considers that Hygiea, the second largest C-type, presents spectral properties similar to those of Ceres (see Appendix A.2 and Figure 7), implying not only a similar formation and thermal evolution, but also contamination by similar enstatite-rich materials. It seems improbable that two of the four largest main belt asteroids were impacted by compositionally similar asteroids, especially if one considers that the two bodies reside in different regions of the asteroid belt (middle belt for Ceres, outer belt for Hygiea). Contamination by IDPs would therefore appear as a more plausible explanation for the presence of enstatite at the surface of Ceres under the assumption that its origin is exogenous. IDP fluxes in this region of the solar system amount to approximately $4 \times 10^{-5}$ particles $/ \mathrm{m}^{2} / \mathrm{s}$ (Grün et al. 2001) implying that a square millimeter is impacted by about $\sim 10^{3}$ particles over $\sim 10^{6}$ years. Considering a typical IDP size of $10-100 \mu \mathrm{m}$, these high fluxes may effectively be compatible with asteroid surfaces being spectrally (at least in the mid-infrared where small particles such as IDPs dominate the emissivity) a mixture of endogenous and exogenous materials. If this hypothesis is correct, enstatite should be found at the surface of compositionally diverse asteroids located in the vicinity of both Ceres and Hygiea. In addition, a contaminating enstatite-rich dust source should exist in the outer belt.

Based on current knowledge, there is one large outer main belt family whose composition is compatible with pyroxenerich IDPs, namely the Themis family (Marsset et al. 2016). Within this family, a recent break up $(<10 \mathrm{Myr})$ has led to the formation of the Beagle family located at the semimajor axis of $a=3.157$ au (Nesvorný et al. 2008). Spitzer Space Telescope observations and numerical modeling have shown that the Beagle family is the most likely source of the prominent $\alpha$ dust band (Nesvorný et al. 2008). Furthermore, metallic asteroids 
located in the vicinity of Ceres and Hygiea show spectral evidence of anhydrous pyroxene-rich dust at their surface (Hardersen et al. 2005), whereas olivine should be observed in case of differentiation. As in the case of Ceres, we suggest an exogenous origin for this dust.

Importantly, an exogenous origin for enstatite questions a similar origin for Ceres, Hygiea, and the remaining C-types such as Eugenia and Themis. As far as Ceres' phyllosilicates are concerned (ammoniated), such a different origin is well supported (De Sanctis et al. 2015): Ceres may have formed in the very outer solar system $(>10 \mathrm{au})$, beyond the ammonia snowline, and may be a close relative of large TNOs such as Orcus. Conversely, enstatite-rich C-types such as Eugenia likely formed in the inner solar system $(<10 \mathrm{au})$, in agreement with model calculations of the dust composition in a stationary protoplanetary solar disk as a function of heliocentric distance (Gail 2004).

\subsection{Constraining the Formation and Evolution of $D<200 \mathrm{~km}$ IDP-like C-type Asteroids}

In this section, we apply our results along with those produced by Takir \& Emery (2012), Vernazza et al. (2015), and Marsset et al. (2016) to formulate a comprehensive scenario for the formation and evolution of IDP-like $D<\sim 200 \mathrm{~km}$ BCG-type asteroids (B, C, Cb, Cg). Spectral similarity in both the 0.4-2.5 $\mu \mathrm{m}$ and $10 \mu \mathrm{m}$ regions (Vernazza et al. 2015; Marsset et al. 2016) suggests similar compositions for the surface of these objects and thus opens the possibility that they accreted from the same building blocks. Both the density (in the $0.8-1.5 \mathrm{~g} \mathrm{~cm}^{-3}$ range; Marchis et al. 2012 and references therein) and the inferred surface composition of these bodies (this work; Vernazza et al. 2015; Marsset et al. 2016) suggest that these building blocks included volatiles (especially water ice) and pyroxene-rich IDP-like material consisting of a minor phase of crystalline pyroxene and a major phase of amorphous silicates. Observations in the $3 \mu \mathrm{m}$ region (Rivkin et al. 2003; Campins et al. 2010; Rivkin \& Emery 2010; Takir \& Emery 2012) have further revealed that the surfaces of these objects consist of water ice and/or anhydrous silicates.

Overall, these observations support a scenario where these objects have suffered limited aqueous alteration and have consequently preserved intact at least part of their primordial outer shell. Indeed amorphous material is very metastable in a hydrothermal environment and rapidly turns into hydrated silicates even at low temperatures (e.g., Nakamura-Messenger et al. 2011). In addition, the outer shell must have been sufficiently thick to prevent either impact excavation of inner hydrated silicates in the course of the solar system history (as suggested in the case of Saturn's satellite Phoebe; CastilloRogez et al. 2012) or collapse of the primordial crust into a subsurface "ocean" during the early thermal evolution (as suggested in the case of Ceres). In support of our analysis, Beauvalet \& Marchis (2014) showed that Eugenia likely possesses a differentiated interior, consisting of a low-porosity $\sim 3 \mathrm{~g} \mathrm{~cm}^{-3}$ dense core ( $<15 \%$ in volume) and a porous icy shell that is $\sim 70-80 \mathrm{~km}$ thick. This internal structure could be consistent with the result of limited early aqueous alteration due to the decay of short lived radionuclides leading to the formation of a small phyllosilicate-rich core (Figure 5). The fact that a significant volume fraction of these bodies has remained unaffected by hydrothermal activity likely implies a late accretion for these bodies. Note that a similar conclusion had been reached for the Themis parent body (Marsset et al. 2016). The internal structure of Eugenia derived by Beauvalet \& Marchis (2014) would naturally explain why the hydrated silicates never reached the surface via impacts and therefore the anhydrous nature of its surface (Figure 5).

In summary, one cannot exclude that IDP-like BCG-type bodies all formed in the same accretional environment, possibly in the giant planet regions, and were scattered across the solar system following, for example, giant planet migrations, as advocated by dynamical models (e.g., Walsh et al. 2011). Their final heliocentric distance would then determine the ability of these bodies to retain water ice on their surfaces in large amounts and/or for a long period of time, in agreement with theoretical estimates of the stability of water ice at the surface/ near surface of main belt asteroids (e.g., Schorghofer 2008). Note that future surveys in the $3 \mu \mathrm{m}$ region will eventually find a phyllosilicate hydration feature in the spectra of some of these objects whose hydrated interiors have been excavated by collisions. This is expected in particular for $D<\sim 100 \mathrm{~km}$ bodies that are believed to be collisional fragments rather than primordial undisrupted bodies (Morbidelli et al. 2009).

\subsection{Cores of IDP-like Asteroids: CI- or Tagish-Lake-like Material?}

In the previous section, we focused our attention on the surface composition of a large $(D \sim 200 \mathrm{~km})$ IDP-like C-type asteroid. So far, spectroscopic surveys in the $3 \mu \mathrm{m}$ region and in the mid-infrared range of these low albedo C-type bodies $\left(p_{\mathrm{v}} \leqslant\right.$ 0.1 ) have mostly focused on the largest objects $(D>\sim 100 \mathrm{~km})$, thus mostly on primordial asteroids that did not suffer a catastrophic disruption (Morbidelli et al. 2009). Thus, it is mostly the composition of the primordial outer shell of these bodies that has been properly characterized so far, and the latter appears to be consistent with anhydrous pyroxene-rich IDP-like material (Vernazza et al. 2015; Marsset et al. 2016). We can extend this inference to $D>100 \mathrm{~km}$ P- and D-type asteroids, whose primordial outer shell mainly consists of a mixture of anhydrous olivine and pyroxene-rich IDP-like material (e.g., Vernazza et al. 2012, 2015).

Concerning the internal compositional structure of these bodies, there are preliminary hints that they may possess a differentiated interior (Beauvalet \& Marchis 2014; Marchis et al. 2014). This differentiation can be well understood as a natural consequence of their early thermal evolution if one assumes that these bodies accreted from a mixture of ice and dust. In this scenario, the core would likely consist of phyllosilicate-rich material.

Extraterrestrial material collections contain a small number of phyllosilicate-rich samples, including CI chondrites, CM chondrites, the atypical Tagish Lake meteorite (e.g., Vernazza et al. 2013 and references therein) and hydrated IDPs. Whereas a large number of potential parent bodies have been identified for CM chondrites (e.g., Vernazza et al. 2016), the same cannot be said for CI chondrites (see Section 11 in Cloutis et al. 2011), Tagish Lake (Vernazza et al. 2013), and hydrated IDPs. The paucity of CI-like bodies is particularly striking since CI falls amount to only one-third of the CM ones. Considering that CM-like asteroids, namely $\mathrm{Ch}$ - and $\mathrm{Cgh}$-types, are routinely observed and represent about one-third of the C-complex asteroids (Rivkin 2012) and that CIs are significantly more friable than CMs, implying a bias against their preservation during atmospheric entry, one would expect CI-like bodies to 


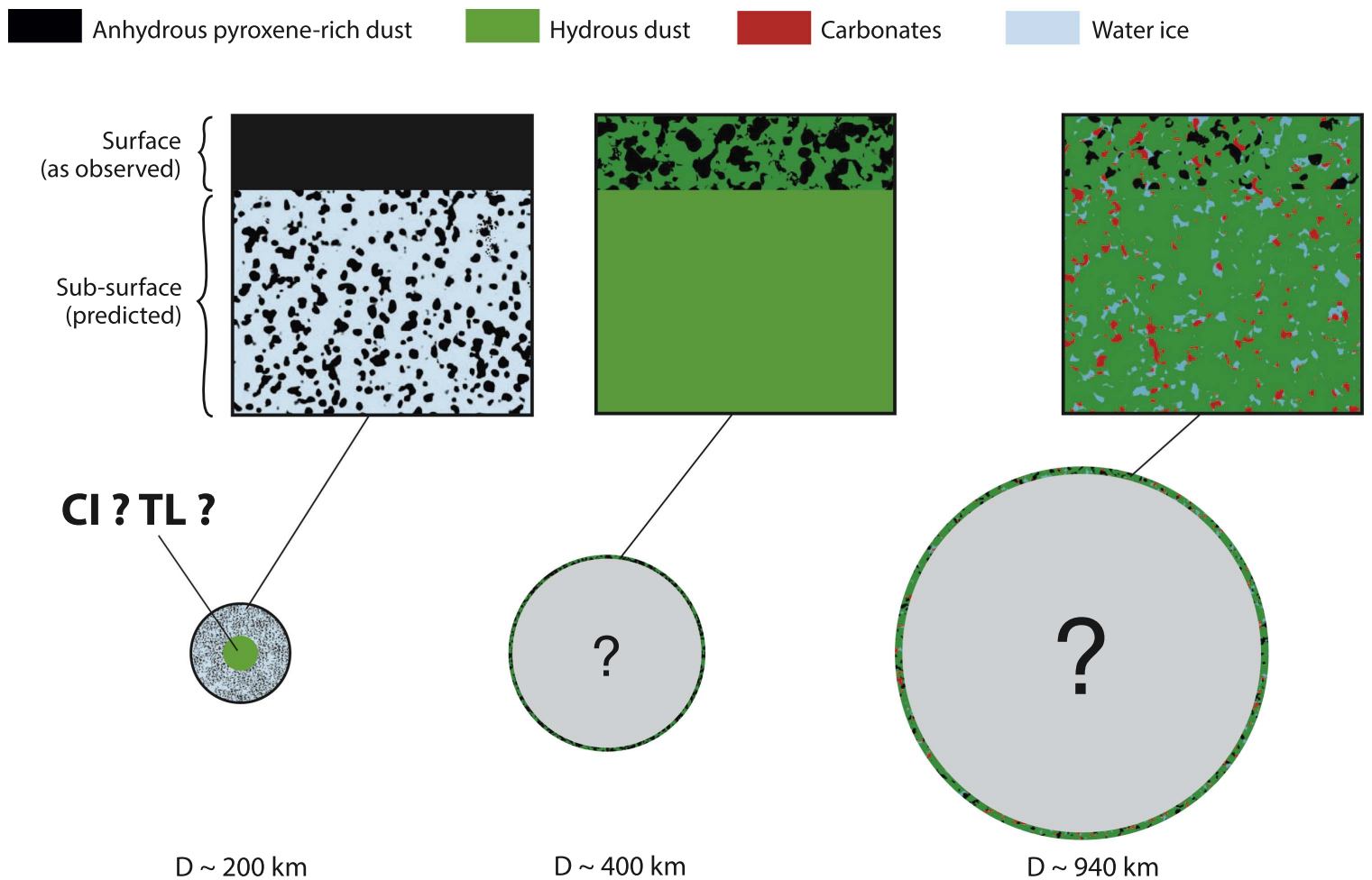

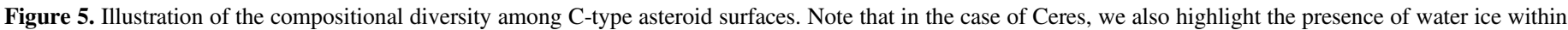

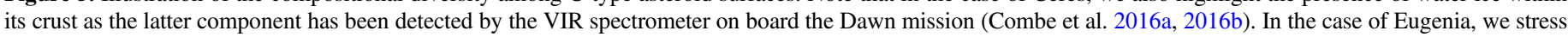

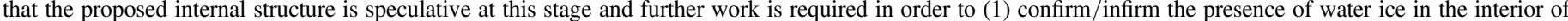

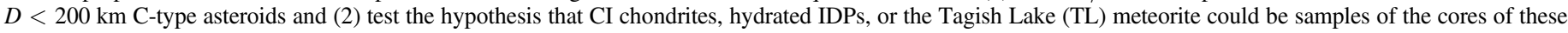
bodies.

be commonly observed as well. However, this is not the case. In a similar register, the paucity of Tagish-Lake-like bodies has already been noticed by Vernazza et al. (2013).

One potential explanation for this paradox could be that CIs, Tagish Lake, and hydrated IDPs are not representative of the original surface material of primordial $D>100 \mathrm{~km}$ asteroids. Instead, these aqueously altered materials may be samples of the cores of IDP-like C-, P-, or D-type $(D>100 \mathrm{~km})$ asteroids. In fact, the water-rich, chondrule-free CIs could potentially represent an aqueously altered, consolidated version of anhydrous IDP-like dust. In other words, CIs could be representative materials of the cores of IDP-like $>100 \mathrm{~km}$ large C-, P-, and D-type bodies that initially accreted from a mixture of ice and dust and where thermal evolution led to the early formation of a phyllosilicate-rich CI-like core (see Section 4.2; e.g., Beauvalet \& Marchis 2014). For similar reasons, both Tagish Lake and hydrated IDPs could also be samples of the cores of IDP-like C-, P-, and D-type bodies.

This conjecture implies that $\mathrm{CI}$-like and Tagish-Lake-like asteroids may have to be searched among smaller, $D<50 \mathrm{~km}$, asteroids that would be leftover fragments of the catastrophic disruption of $D>100 \mathrm{~km}$ IDP-like C-, P-, and D-type bodies. In this respect, collisional families offer a great opportunity to investigate the spectral properties and thus composition of these cores. The Eurybates family, the only well-identified collisional family among the Jupiter Trojans (P/D types), whose parent bodies were estimated to be in the $D \sim 110-130 \mathrm{~km}$ size range (Brož \& Rozehnal 2011), is particularly relevant here. Spectroscopic observations of the family members have revealed a great spectral variety (Fornasier et al. 2007; De Luise et al. 2010), with about half of the family members being C-types and the remaining objects being P-types. Considering that the largest Jupiter Trojans are either P- or D-type asteroids, these observations could suggest that the cores of P-/D-type asteroids may consist of C-type-like material. Since CI chondrites possess mostly C-type-like spectral properties, this opens the possibility that $\mathrm{CI}$ chondrites could be samples of the cores of P/D-type asteroids. Note that is seems quite unlikely that space-weathering processes could be at the origin of the slope variation within the family (i.e., Cs and Ps) given that the surface age should be roughly the same for all objects. If there was only one C-type, one could argue for a recent resurfacing event but in the present case the similar frequency of $\mathrm{Cs}$ and Ps tends to exclude the space-weathering hypothesis.

These preliminary ideas lead to new questions to be addressed in future research.

1. Could CI chondrites and Tagish Lake represent samples of the cores of $D>100 \mathrm{~km}$ IDP-like C-, P-, and D-type bodies? Or would hydrated IDPs be more relevant analogs?

2. What is the expected compositional difference between the core of an IDP-like C-type (e.g., Eugenia) and the core of an IDP-like D-type (e.g., Hektor) and what are their respective extraterrestrial analogs (if any)?

\section{CONCLUSION}

Spectroscopic observations in the 5-35 $\mu \mathrm{m}$ range of Ceres and Eugenia enabled a deeper investigation of the compositional diversity among C-type asteroids. Our derived compositions appear not only consistent with previous interpretations 


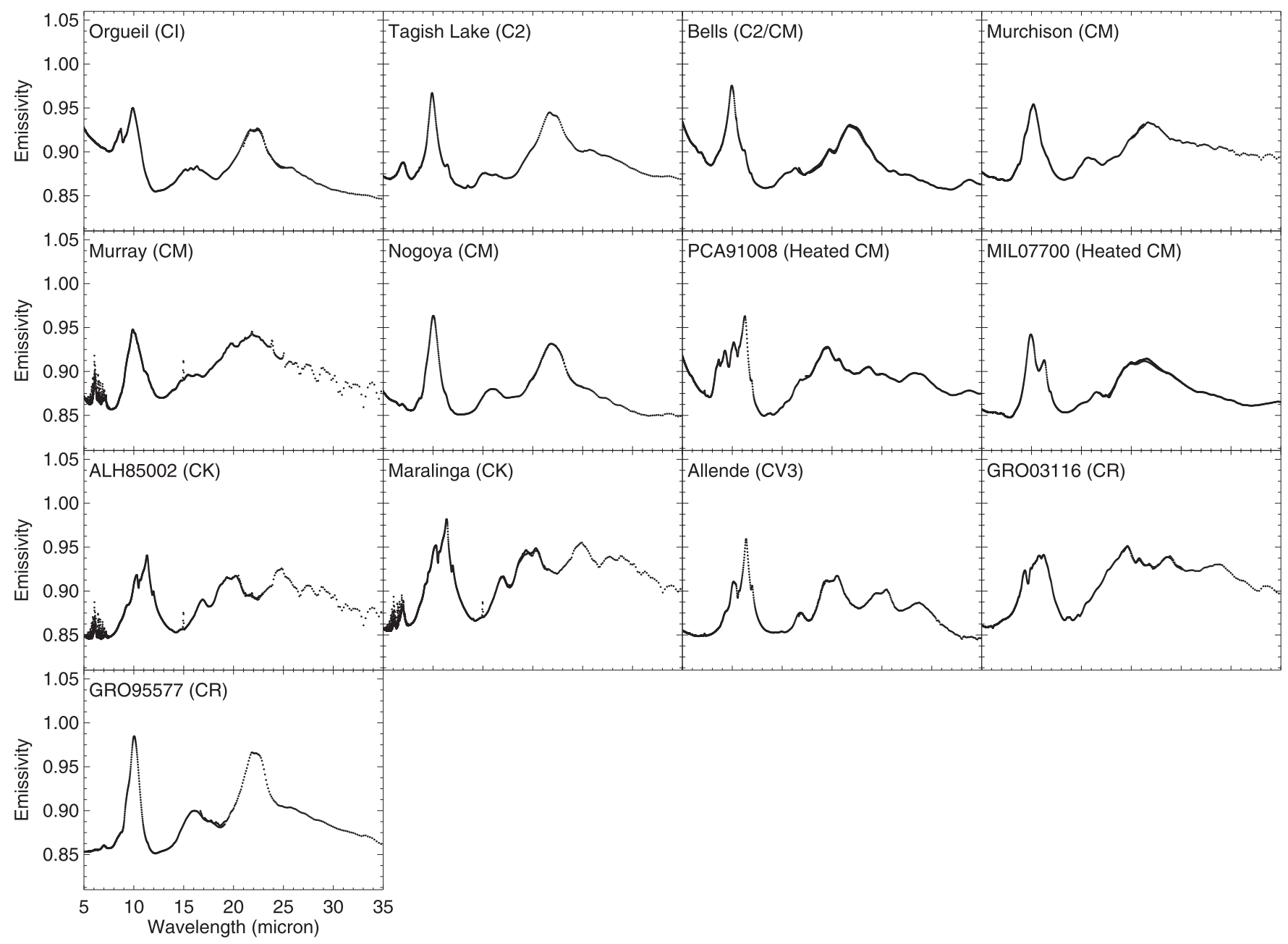

Figure 6. Mid-infrared spectral properties of carbonaceous chondrite meteorites collected over the 5-35 $\mu \mathrm{m}$ range. For each meteorite, a pellet was synthesized using $1.0 \mathrm{mg}$ of meteorite dust dispersed in $300 \mathrm{mg}$ of ultrapure $\mathrm{KBr}$. The transmission Mid-IR $\left(2-25 \mu \mathrm{m}\right.$, resolution $\left.2 \mathrm{~cm}^{-1}\right)$ spectra of the pellets were measured at Institut de Planétologie et d'Astrophysique de Grenoble (France; Beck et al. 2014), whereas the far-IR spectra were collected with the SMIS beamline (Dumas et al. 2006) of the synchrotron SOLEIL (France).

derived from spectral analyses performed at shorter wavelengths (in the $3 \mu \mathrm{m}$ region in particular) but they are also complementary. Both the "wet" and "dry" nature of the surfaces of, respectively, Ceres and Eugenia could be revealed in the mid-infrared range. In addition, our investigation allowed the detection and subsequent characterization of the anhydrous silicate composition present at the surfaces of both objects. Specifically, enstatite is likely present on the surface of Ceres at a level of at least 20vol.\% mixed with products of aqueous alteration (Dawn VIR observations could only detect the latter), whereas both enstatite and amorphous silicates are the main surface components of Eugenia. Furthermore, our analysis confirms earlier suggestions that carbonaceous chondrite meteorites are poor analogs for the refractory material present on these objects. Importantly, the presence of enstatite at the surface of Ceres is striking and unexpected. The most plausible explanation for the origin of this component is an exogenous delivery of pyroxene-rich dust possibly coming from the Beagle family. This scenario is compatible with, yet does not imply, an outer solar system origin for Ceres, as indicated by the detection of ammoniated phyllosilicates on its surface by the Dawn mission (De Sanctis et al. 2015).
For the smaller $D<\sim 200 \mathrm{~km}$ C-types, we propose that these bodies accreted from the same building blocks, namely chondritic porous, pyroxene-rich (and amorphous-rich) IDPs and volatiles (mostly water ice), and that a significant volume fraction of these bodies has remained unaffected by hydrothermal activity, likely implying a late accretion for these bodies. In addition, their current heliocentric distance may best explain the presence or absence of water ice at their surfaces. We also introduce a new hypothesis regarding the composition of the cores of these bodies: hydrated extraterrestrial materials such as CI chondrites, the Tagish Lake meteorite, and hydrous IDPs appear to be plausible candidates.

Finally, it is important to stress that the future of asteroid science would greatly benefit from a space-based observatory dedicated to the observations of large- $(D>100 \mathrm{~km})$ and medium-sized $(20 \mathrm{~km}<D<100 \mathrm{~km})$ asteroids in the $\sim 5-25 \mu \mathrm{m}$ spectral range. Data sets collected with ISO and Spitzer for these asteroids exist only for a few objects $(<30)$. That type of observation cannot be performed with the James Webb Space Telescope because objects with $D>20 \mathrm{~km}$ will be too bright for that telescope (Rivkin et al. 2016). 

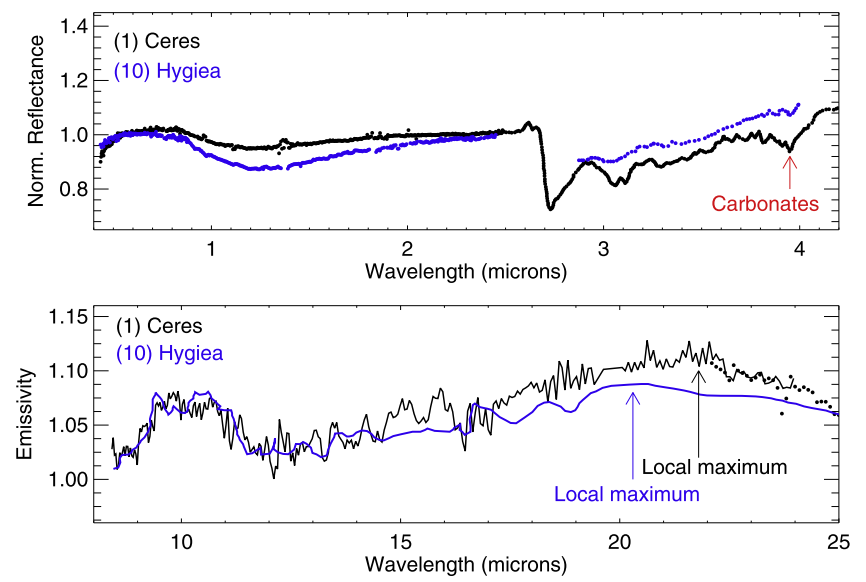

Figure 7. Comparison between the visible near- and mid-infrared spectral properties of (1) Ceres and (10) Hygiea. The data were retrieved from http:// smass.mit.edu/ and from the following papers: Takir \& Emery (2012), Barucci et al. (2002), and De Sanctis et al. (2015). Whereas the two asteroids possess very similar spectral properties, there are some noticeable differences between the two spectra: (1) the $3.06 \mu \mathrm{m}$ band attributed to ammoniated phyllosilicates (De Sanctis et al. 2015) is much deeper in the spectrum of Ceres than in the Hygiea one, (2) the $3.95 \mu \mathrm{m}$ band due to the presence of carbonates is only unambiguously identified in the spectrum of Ceres, and (3) the position of the $20 \mu \mathrm{m}$ emissivity peak of Hygea is located at shorter wavelength with respect to that of Ceres.

We thank the referee for pertinent and constructive remarks. This work is based on observations made with the NASA/DLR Stratospheric Observatory for Infrared Astronomy (SOFIA). SOFIA is jointly operated by the Universities Space Research Association, Inc. (USRA), under NASA contract NAS2-97001, and the Deutsches SOFIA Institut (DSI) under DLR contract 50 OK 0901 to the University of Stuttgart. We warmly thank Melody Didier for producing Figure 5's artwork.

\section{APPENDIX}

\section{A.1. Laboratory Data for Meteorites}

Here, we present new mid-infrared laboratory emissivity spectra of 13 carbonaceous chondrite meteorites collected over the 16.6-35 $\mu \mathrm{m}$ range. These new spectra complement those acquired by Beck et al. (2014) over the $2-25 \mu \mathrm{m}$ range for the same samples. Accordingly, a detailed description of the sample preparation can be found in Beck et al. (2014).

New mid-IR micro-analysis was performed at the SMIS beamline (Dumas et al. 2006) of the synchrotron SOLEIL (France). We used a NicPlan microscope (x32 objective) coupled to a NEXUS 6700 FTIR spectrometer (Thermo Fisher) operating in transmission, in a similar configuration previously used to analyze cometary dust (see Brunetto et al. 2011 for more details). The analyzed spot diameter was $20-30 \mu \mathrm{m}$ on the samples, so that several positions were analyzed and spectra were averaged to increase the signal-to-noise ratio. Spectra were collected in the $600-285 \mathrm{~cm}^{-1}(16.6-35 \mu \mathrm{m})$ spectral range because the $\mathrm{KBr}$ matrix is poorly transparent for wavelength larger than $\sim 35 \mu \mathrm{m}$. In the $25-35 \mu \mathrm{m}$ spectral range, a correction was applied to take into account the small $\mathrm{KBr}$ absorption based on the knowledge of the $\mathrm{KBr}$ optical constants (Palik 1985) and the thickness of the pellets, we estimated the contribution of the $\mathrm{KBr}$ to the absorption and subtracted it from the measured spectra (error bars are $<5 \%$ in the $25-30 \mu \mathrm{m}$ range, and $<10 \%$ in the $30-35 \mu \mathrm{m}$ range). The spectra were finally adjusted and combined to the Beck et al. spectra by using the overlapping spectral range, and they are presented in Figure 6.

\section{A.2. Similarities and Differences in Composition between Ceres and Hygiea}

(10) Hygiea is the only known main belt asteroid that possesses spectral properties nearly similar to (1) Ceres in the $0.4-2.5 \mu \mathrm{m}$ range, in the $3 \mu \mathrm{m}$ range, and in the $10 \mu \mathrm{m}$ and $20 \mu \mathrm{m}$ regions (see Figure 7). There are, however, three noticeable spectral differences between the two objects. The first is the absence of a carbonate band around $\sim 3.95 \mu \mathrm{m}$ in the spectrum of Hygiea. The second is a shift in the $20 \mu \mathrm{m}$ peak found at Hygiea toward shorter wavelengths $(\sim 20 \mu \mathrm{m})$ with respect to Ceres $(\sim 22 \mu \mathrm{m})$. The third is a shallower depth of the $\sim 3.06 \mu \mathrm{m}$ feature in the spectrum of Hygiea with respect to Ceres. All of these differences indicate that the surface of Hygiea contains less hydrated minerals than Ceres; conversely, they suggest that anhydrous silicates are more abundant at the surface of Hygiea than Ceres. The mid-infrared range supports this assumption particularly well since anhydrous silicates such as amorphous and crystalline pyroxene possess emissivity peaks around $\sim 20 \mu \mathrm{m}$, whereas phyllosilicates peak around $\sim 22 \mu \mathrm{m}$.

\section{REFERENCES}

Ammannito, E., DeSanctis, M. C., Ciarniello, M., et al. 2016, Sci, 353, aaf4279 Ammannito, E., de Sanctis, M. C., Palomba, E., et al. 2013, Natur, 504, 122 Avdellidou, C., Price, M. C., Delbo, M., Ioannidis, P., \& Cole, M. J. 2016, MNRAS, 456, 2957

Barucci, M. A., Dotto, E., Brucato, J. R., et al. 2002, Icar, 156, 202

Beauvalet, L., \& Marchis, F. 2014, Icar, 241, 13

Beck, P., Quirico, E., Garenne, A., et al. 2014, Icar, 229, 263

Bland, P. A., Cressey, G., \& Menzies, O. N. 2004, M\&PS, 39, 3

Bradley, J. P. 1994, Sci, 265, 925

Brož, M., \& Rozehnal, J. 2011, MNRAS, 414, 565

Brunetto, R., Borg, J., Dartois, E., et al. 2011, Icar, 212, 896

Burbine, T. H. 2014, in Asteroids. Planets, Asteriods, Comets and The Solar System, Vol. 2 of Treatise on Geochemistry, ed. A. M. Davis (2nd ed.; Amsterdam: Elsevier), 365

Campins, H., Hargrove, K., Pinilla-Alonso, N., et al. 2010, Natur, 464, 1320 Castillo-Rogez, J. C. 2011, Icar, 215, 599

Castillo-Rogez, J. C., Johnson, T. V., Thomas, P. C., et al. 2012, Icar, 219, 86 Castillo-Rogez, J. C., \& McCord, T. B. 2010, Icar, 205, 443

Chamberlain, M. A., Lovell, A. J., \& Sykes, M. V. 2009, Icar, 202, 487

Clark, B. E., Ziffer, J., Nesvorny, D., et al. 2010, JGR, 115, E06005

Cloutis, E. A., Hiroi, T., Gaffey, M. J., Alexander, C. M. O. 'D., \& Mann, P. 2011, Icar, 212, 180

Combe, J.-Ph., McCord, T. B., Tosi, F., et al. 2016a, in 47th Lunar and Planetary Science Conf. Detection of H2O-Rich Materials on Ceres by the Dawn Mission, Contribution No. 1903, 1820

Combe, J.-Ph., McCord, T. B., Tosi, F., et al. 2016b, Sci, 353, aaf3010

Daly, R. T., \& Schultz, P. H. 2015, GeoRL, 42, 7890

Daly, R. T., \& Schultz, P. H. 2016, Icar, 264, 9

De Luise, F., Dotto, E., Fornasier, S., et al. 2010, Icar, 209, 586

DeMeo, F. E., Binzel, R. P., Slivan, S. M., \& Bus, S. J. 2009, Icar, 202, 160

DeMeo, F. E., \& Carry, B. 2013, Icar, 226, 723

De Sanctis, M. C., Ammannito, E., Raponi, A., et al. 2015, Natur, 528, 241

De Sanctis, M. C., Raponi, A., Ammannito, E., et al. 2016, Natur, 536, 54

Dumas, P., Polack, F., Lagarde, B., et al. 2006, InPhT, 49, 152

Emery, J. P., Cruikshank, D. P., \& van Cleve, J. 2006, Icar, 182, 496

Formisano, M., Federico, C., De Angelis, S., De Sanctis, M. C., \& Magni, G. 2016, MNRAS, 463, 520

Fornasier, S., Dotto, E., Hainaut, O., et al. 2007, Icar, 190, 622

Fu, R. R., Ermakov, A. I., Marchi, S., et al. 2016, in 79th Annual Meeting of the Meteoritical Society, Thermal Evolution and Fluid Flow in Planetesimals Inferred from Dawn Mission Observations of Ceres, 6107

Gail, H. P. 2004, A\&A, 413, 571 
Grün, E., Baguhl, M., Svedhem, H., \& Zook, H. A. 2001, in Interplanetary Dust, In situ Measurements of Cosmic Dust, ed. E. Grün et al. (Berlin: Springer), 295

Hardersen, P. S., Gaffey, M. J., \& Abell, P. A. 2005, Icar, 175, 141

Harris, A. W. 1998, Icar, 131, 291

Hendrix, A. R., Vilas, F., \& Li, J.-Y. 2016, GRL, 43, 8920

Hiroi, T., Pieters, C. M., Zolensky, M. E., \& Lipschutz, M. E. 1993, Sci, 261, 1016

Hiroi, T., Zolensky, M. E., Pieters, C. M., \& Lipschutz, M. E. 1996, M\&PS, 31,321

Ishii, H. A., Bradley, J. P., Dai, Z. R., et al. 2008, Sci, 319, 447

Izawa, M. R. M., King, P. L., Flemming, R. L., Peterson, R. C., \& McCausland, P. J. A. 2010, JGR, 115, E07008

King, A. J., Schofield, P. F., \& Russell, S. S. 2015, in 78th Annual Meeting of the Meteoritical Society, LPI Contribution No. 1856, Thermal Alteration of CI and CM Chondrites: Mineralogical Changes and Metamorphic Temperatures, 5212

Lantz, C., Clark, B. E., Barucci, M. A., \& Lauretta, D. S. 2013, A\&A, 554, 7

Li, J.-Y., Reddy, V., Nathues, A., et al. 2016, ApJL, 817, L22

Marchis, F., Durech, J., Castillo-Rogez, J., et al. 2014, ApJL, 783, L37

Marchis, F., Enriquez, J. E., Emery, J. P., et al. 2012, Icar, 221, 1130

Marsset, M., Vernazza, P., Birlan, M., et al. 2016, A\&A, 586, A15

McAdam, M. M., Sunshine, J. M., Howard, K. T., \& McCoy, T. M. 2015, Icar, 245,320

McCord, T. B., Li, J.-Y., Combe, J.-P., et al. 2012, Natur, 491, 83

McCord, T. B., \& Sotin, C. 2005, JGR, 110, E05009

Merouane, S., Djouadi, Z., \& Le Sergeant d'Hendecourt, L. 2014, ApJ, 780,174

Milliken, R. E., \& Rivkin, A. S. 2009, NatGe, 2, 258

Min, M., Hovenier, J. W., \& de Koter, A. 2003, A\&A, 404, 35

Morbidelli, A., Bottke, W. F., Nesvorný, D., \& Levison, H. F. 2009, Icar, 204, 558

Mueller, T. G., \& Lagerros, J. S. V. 1998, A\&A, 338, 340

Nakamura-Messenger, K., Clemett, S. J., Messenger, S., \& Keller, L. P. 2011, M\&PS, 46, 843

Nesvorný, D., Bottke, W. F., Vokrouhlický, D., et al. 2008, ApJL, 679, L143
Neveu, M., \& Desch, S. J. 2015, GeoRL, 42, 10197

Palik, E. D. 1985, Handbook of Optical Constants of Solids (New York: Academic Press)

Palomba, E., Longobardo, A., De Sanctis, M. C., et al. 2015, Icar, 258, 120

Park, R. S., Konopliv, A. S., Bills, B., et al. 2016, in 47th Lunar and Planetary Science Conf., LPI Contribution No. 1903, Gravity Science Investigation of Ceres from Dawn, 1781

Petit, S., Righi, D., Madejova, J., \& Decarreau, A. 1999, Clay Minerals, 34, 543

Poulet, F., Ruesch, O., Langevin, Y., \& Hiesinger, H. 2015, Icar, 253, 364

Reddy, V., Le Corre, L., O'Brien, D. P., et al. 2012, Icar, 221, 544

Rivkin, A. S. 2012, Icar, 221, 744

Rivkin, A. S., Davies, J. K., Johnson, J. R., et al. 2003, M\&PS, 38, 1383

Rivkin, A. S., \& Emery, J. P. 2010, Natur, 464, 1322

Rivkin, A. S., Marchis, F., Stansberry, J. A., et al. 2016, PASP, 128, 018003

Rivkin, A. S., Thomas, C. A., Howell, E. S., \& Emery, J. P. 2015, AJ, 150, 198

Rivkin, A. S., Volquardsen, E. L., \& Clark, B. E. 2006, Icar, 185, 563

Ruesch, O., Hiesinger, H., De Sanctis, M. C., et al. 2014, JGRE, 119, 2078

Russell, C. T., Raymond, C. A., Ammannito, E., et al. 2016, Sci, 353, 1008

Sandford, S. A., \& Walker, R. M. 1985, ApJ, 291, 838

Schorghofer, N. 2008, ApJ, 682, 697

Takir, D., \& Emery, J. P. 2012, Icar, 219, 641

Turrini, D., Combe, J.-P., McCord, T. B., et al. 2014, Icar, 240, 86

Turrini, D., Svetsov, V., Consolmagno, G., Sirono, S., \& Pirani, S. 2016, Icar, 280,328

Vernazza, P., Fulvio, D., Brunetto, R., et al. 2013, Icar, 225, 517

Vernazza, P., Lamy, P., Groussin, O., et al. 2011, Icar, 216, 650

Vernazza, P., Marsset, M., Beck, P., et al. 2015, ApJ, 806, 204

Vernazza, P., Marsset, M., Beck, P., et al. 2016, AJ, 152, 54

Vernazza, P., Delbo, M., King, P. L., et al. 2012, Icar, 221, 1162

Vilas, F., \& Gaffey, M. J. 1989, Sci, 246, 790

Vilas, F., Larson, S. M., Hatch, E. C., \& Jarvis, K. S. 1993, Icar, 105, 67

Walsh, K. J., Morbidelli, A., Raymond, S. N., O’Brien, D. P., \& Mandell, A. M. 2011, Natur, 475, 206

Yang, B., \& Jewitt, D. 2010, AJ, 140, 692 